\title{
Comparación del modelo paliativo y el modelo geriátrico en la calidad de muerte en una población con demencia avanzada en un programa de visita domiciliar
}

Comparison of a palliative model and geriatric model in the quality of death in a population of a home visitation program with advanced dementia

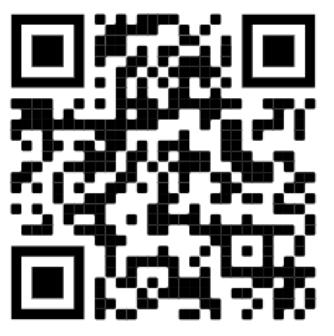

1Dr. José Ernesto Picado Ovares Hospital nacional de geriatría y gerontología Dr. Raúl Blanco Cervantes, San José, Costa Rica

https://orcid.org/0000-0001-5096-0980

${ }^{2}$ Bach. Fabián de Jesús Parra Parra Investigador independiente, San José, Costa Rica

(iD) https://orcid.org/0000-0002-7270-9153.

${ }^{3}$ Bach. Jimena Ruiz Rivera Investigadora independiente, San José, Costa Rica

(Dhttps://orcid.org/0000-0002-4223-7271

Recibido

$23 / 12 / 2020$
Corregido

$10 / 01 / 2021$
Aceptado

07/02/2021

\section{RESUMEN}

Introducción: los adultos mayores con demencia avanzada son un grupo de población en crecimiento y que tienen necesidades particulares. No está claro cuál modelo de atención pueda generar una mejor calidad de muerte. Objetivo: comparar el impacto del modelo de tratamiento paliativo contra el geriátrico en la calidad de muerte en los pacientes con demencia avanzada. Metodología: se aplicó el Quality of Dying and Death Questionnaire a los cuidadores de los pacientes que habían fallecido en los años del 2016 al 2018 y que el diagnóstico de ingreso al programa fuera el de demencia avanzada. Se generaron dos grupos dependiendo del programa que realizó la atención en el domicilio, ya sea el programa de cuidados paliativos o el de geriatría. Se realiza un análsis descriptivo de ambos grupos. Se evalúa el total general dentro de las secciones del cuestionario, así como, cada uno de los apartados que las conforman. Resultados: se registraron 162 entrevistas, 125 del cuidadores del programa geriátrico y 37 del de paliativos. Los pacientes murieron generalmente con dolor controlado, con poca conciencia de lo que pasaba alrededor, respirando cómodamente, tranquilos, sin miedo a morir, sin preocupaciones por ser una carga para la familia, pasando tiempo con sus amigos y recibiendo afecto de sus seres

\footnotetext{
${ }^{1}$ Médico Especialista en geriatría y gerontología, graduado de la Universidad de Costa Rica (UCR). Master en Cuidados Paliativos, Universidad de Valladolid, España. Cod. MED7413,correo: neto79es@yahoo.es

2 Bachiller en estadística, graduado dela Universidad de Costa Rica (UCR), correo: Fabianpp2412@gmail.com

${ }^{3}$ Bachiller en estadística, graduada de la Universidad de Costa Rica (UCR), correo: Jruizrivera1@gmail.com
} 
queridos. Los gastos, arreglos funerarios y la visita de un concejero espiritual se dieron de forma adecuada en ambos grupos. El índice de calidad de muerte y la satisfacción en la atención fue bastante alto. No hubo diferencias significativas entre ambos grupos. Conclusión: el grado de satisfacción en la atención por parte de ambos grupos fue muy bueno sin encontrarse diferencias significativas. Se debe realizar mayores investigaciones en esta población para ofrecer un modelo óptimo apropiado para sus necesidades.

PALABRAS CLAVE: demencia; envejecimiento; geriatría; atención dirigida al paciente; visita domiciliaria.

\section{ABSTRACT}

Introduction: older adults with advanced dementia are a growing population group with particular needs. It is unclear which model of care can generate a better quality of death. Objective : to Compare the impact of the palliative treatment model against geriatrics on the quality of death in patients with advanced dementia.Methodology: the Quality of Dying and Death Questionnaire was applied to caregivers of patients who had died in the years 2016 to 2018 and that the diagnosis of entry into the program was that of advanced dementia. Two groups were generated depending on the program that performed the homecare, either the palliative care program or the geriatrics program. A descriptive analysis of both groups is performed. The overall total of the questionnaire is evaluated, as well as each of the constituent parts. Results: there were 162 interviews, 125 from the caregivers of the geriatric program and 37 from the palliative program. Patients died usually with controlled pain, with little awareness of what was going on around, breathing comfortably, calmly, without fear of dying, without worries about being a burden to the family, spending time with their friends and receiving affection from their loved ones. Expenses, funeral arrangements, and the visit of a spiritual advisor were given appropriately in both groups. The death quality rate and attention satisfaction were quite high. There were no significant differences between the two groups. Conclusion: the degree of satisfaction in attention on the part of both groups was very good without significant differences. Further research should be done in this population to provide an optimal model appropriate for your needs.

KEY WORDS: dementia; aging; geriatrics; patient care; home visit.

\section{INTRODUCCIÓN}

El grupo poblacional de los adultos mayores será el grupo de mayor crecimiento a nivel mundial. Para el año 2050, la proporción de los habitantes mayores de 60 años se duplicará, llegando a un $22 \%$ de la población mundial (1).

Estos cambios en la pirámide poblacional impactarán tanto la prevalencia como la incidencia de las enfermedades no oncológicas asociadas a la edad, donde se pueden incluir los síndromes demencias. Según la Alzheimer's Disease International (2) para ese año, 131,5 millones de adultos mayores tendrán el diagnóstico de demencia, siendo en los países en vías de desarrollo donde esta enfermedad aumentará de forma más acelerada.

Los cuidados paliativos se encargan de aliviar el sufrimiento de personas y familias que se enfrentan a una enfermedad terminal. De acuerdo con Van der Steen (므), la demencia es una de estos padecimientos y comparte similitudes con otras enfermedades. Sin embargo, posee características propias $(4,5)$ que hacen su abordaje distinto y sumamente complejo $(\underline{6}, \underline{7})$.

La Asociación Europea de Cuidados Paliativos publicó recientemente un consenso de expertos que busca definir los 
principios básicos para una adecuada atención paliativa dirigida a los pacientes con este síndrome, enfocándose en respetar la dignidad, la autonomía y en mejorar la calidad de vida y de muerte del paciente (으). Sin embargo, se evidencia en la literatura la dificultad y la escasez de información para poder descifrar la mejor alternativa de atención paliativa para este grupo poblacional pues, como señala Brian ( $\underline{9})$ depende mucho del contexto social donde se presente.

Generalmente, en latinoamérica, estos pacientes no reciben el apoyo necesariol lo que se refleja en una atención poco satisfactoria (10) con una peor calidad de muerte y a una relación costo/beneficio desfavorable (11-13).

Es por esto por lo que se hace vital generar investigación de calidad relacionada con los modelos de atención que se ajusten a la realidad nacional y que ofrezcan mejor calidad de muerte para los pacientes.

La Unidad de Atención Comunitaria Geriátrica (ACG) del Hospital Nacional de Geriatría y Gerontología, Doctor Raúl Blanco Cervantes de Costa Rica (HNGGDRBC) se fundó en 1996. Desde entonces realiza visitas por un equipo interdisciplinario a pacientes con enfermedades crónicas degenerativas terminales, para así ofrecer la atención hospitalaria a nivel del domicilio. En la TABLA 1 se resume el estado actual del servicio de la ACG y los servicios ofrecidos (14).

El objetivo de esta investigación es comparar, en los pacientes con demencia avanzada, el impacto del modelo de tratamiento paliativo contra el modelo de atención geriátrico en la calidad de muerte utilizando el Quality of Dying and Death Questionnairevalidado en español (QODDES) (15), para valorar cuál es el más adecuado para implementar en esta población particular. La escala de calidad QODD-ES es ampliamente reconocida y validada al español, y se utiliza para medir la calidad de muerte en la población de cuidados paliativos. Cuenta con 3 módulos (15).

- El primer módulo, titulado "Calidad de muerte", cuenta con 4 posibles opciones para las respuestas y pregunta sobre aspectos relacionados con la experiencia de los últimos 7 días de vida del paciente.

- El segundo módulo se valora por medio de una pregunta de opción dicotómica (sí o no) si las necesidades básicas de los adultos mayores fueron cubiertas antes de morir y por último.

- El tercer módulo busca recolectar la información por medio de 12 preguntas que piden valorar en una escala del 0 al 100 la calidad de muerte; así se construye finalmente, utilizando solamente este último módulo, el índice de calidad de muerte (ICM).

Para este estudio, se agregó un cuarto módulo que buscaba valorar algunas variables de cada programa que pudieran explicar las diferencias eventualmente encontradas en la calidad de muerte entre cada programa.

\section{MÉTODO}

Para valorar la calidad de muerte de los pacientes con demencia avanzada, se aplicó el cuestionario sobre calidad de muerte QODD-ES.

Este cuestionario fue aplicado por los investigadores telefónicamente a los cuidadores entre el 2 al 9 de octubre del 2019. Los entrevistados se identificaron utilizando la base de datos de las sesiones de ingreso del servicio de ACG. Las 
personas entrevistadas eran cuidadores de pacientes que cumplían todas las siguientes características:

- Pacientes que ingresaron al programa en los años del 2016 al 2018.

- Su diagnóstico de ingreso fue el de "demencia avanzada "con una escala de Global Dementia Scale 7 (GDS 7).

- Pacientes que hayan fallecido entre el año 2016 al 2018.

Los cuidadores seleccionados se subdividieron en dos grupos: "geriátricos" y "paliativos", de acuerdo con el programa que realizó las visitas a los pacientes que ellos atendían al momento del fallecimiento.

El cuestionario sobre calidad de muerte utilizado (QODD-ESP-12) recolecta la información general relacionada con los últimos momentos del adulto mayor. Sin embargo, tomando en cuenta el público meta al cuál se le iba a aplicar el cuestionario, para la presente investigación, se realizaron leves modificaciones de redacción y estructura con la intención de que las preguntas fueran más claras al momento de la entrevista. Se realizaron 3 intentos distintos en los casos donde no hubo respuesta a la primera llamada.Las respuestas obtenidas en las cuatro partes del cuestionario- encuesta de "Satisfacción del Servicio de Atención Comunitaria Geriátrica", se analizaron por medio de análisis descriptivos, separando según el programa de atención recibido. Se evalúa un total general dentro de las cuatro secciones del cuestionario, así como, cada uno de los apartados que las conforman.

Una vez que se tienen los análisis descriptivos, se realiza un estudio más a profundidad para evaluar si existen diferencias significativas entre los valores observados del programa crónico contra el paliativo.

Se crean intervalos de confianza al 95\% para las proporciones, utilizando el método de Wald a partir de la distribución multinomial. En el caso de los intervalos de confianza para la media, se aproximan a la distribución normal, para una desviación estándar poblacional desconocida y estimada mediante los datos observados.

Se busca contrastar como hipótesis nula que los porcentajes observados de crónicos y paliativos dentro de cada ítem, tienen diferencias nulas. Para realizar lo anteriormente mencionado, se emplea una significancia del $5 \%$ en la prueba realizada; por medio de esto, lograr observar si hay diferencias significativas dentro de los dos grupos de programas analizadosFinalmente, para el cálculo del Índice de Calidad de Muerte (ICM) se utiliza únicamente el tercer módulo del cuestionario, que establece en una escala de 0 a 10 cómo una experiencia en específico afectó los últimos momentos de la vida del paciente, según la percepción del cuidador. El cálculo se realiza de la siguiente manera:

$\mathrm{ICM}=$ (Suma de valores de la escala)/(Número de preguntas respondidas) $\mathrm{x} 10$

El ICM comprende el rango de 0 a 100, donde 0 indica una muy mala experiencia al morir y 100 una muy buena experiencia al morir, según los aspectos evaluados. Para determinar si las diferencias en el ICM entre ambos programas de atención domiciliaria son estadísticamente significativas, se ajusta un modelo de regresión utilizando como variable dependiente el ICM y como variable independiente la variable programa (geriátrico o paliativo), y se contrasta la 
hipótesis que establece que las medias de las poblaciones en ambos programas son iguales mediante una prueba $F$ en un análisis de varianza.

Por último, al tratarse de un tema sensible en las entrevistas, se pidió inicialmente el consentimiento informado a los participantes y se les ofreció atención psicológica especializada a los cuidadores que así los solicitaban o cuando se detectara por parte de los entrevistados la necesidad de esta atención.

\section{RESULTADOS}

Para este estudio, se registró un total de 240 adultos mayores que pertenecieron al programa de ACG durante el año 2016 y 2018 que cumplieron con los criterios de ingreso. De estos, 190 pertenecían al programa de geriatría domiciliar y 50 del programa de paliativos domiciliares. Luego de 4 intentos, se lograron obtener los datos de las entrevistas del $67 \%$, lo que representa a 162 entrevistas.

El programa de geriatría obtuvo un porcentaje de respuesta de $66 \%$ y el de paliativos del $72 \%$, lo que representan 125 y 37 entrevistas respectivamente.

Las características principales de los pacientes y los cuidadores de cada grupo se muestran en el TABLA 2, las cuales son similares y han sido analizados en estudios previos en esta población (16).

En CUADRO 1 se aprecian los resultados del primer módulo del cuestionario. No hubo diferencias significativas entre ambos grupos, tanto en el total de los datos como al analizarlos cada pregunta de forma individual.

Como se aprecia en el cuadro respectivo, según los cuidadores directos, los pacientes murieron generalmente con dolor controlado, con poca conciencia de lo que pasaba alrededor, respirando cómodamente, tranquilos, sin miedo a morir, sin preocupaciones por ser una carga para la familia, pasando tiempo con sus amigos y recibiendo afecto de sus seres queridos. Como se muestra en el CUADRO 2, en relación a la segunda parte del cuestionario, relacionado con las necesidades básicas, se documenta que en la mayoría de los casos en ambos grupos, los gastos y arreglos funerarios, la visita de un concejero espiritual se dieron de forma adecuada en la mayoría de los entrevistados de ambos grupos. Por otro lado, fueron muy pocos los casos donde se utilizó las "máquinas para respirar". En la tercera parte del cuestionario, relacionado específicamente al índice de calidad de muerte, se documenta en el CUADRO 3 un ICM bastante alto. Solamente en la pregunta 4 se presentó una diferencia estadísticamente significativa. El cuarto y último modulo se meustran en el CUADRO 4 donde se documenta que en general la satisfacción entre ambos grupos es muy buena.

\section{DISCUSIÓN}

Al analizar los resultados del CUADRO 1, en general, los porcentajes reflejan una buena calidad de muerte de ambos programas en todas las catergorías. Estos resultados son similares a estudios previos, como el elaborado por Zimmermann (16).

En relación con los resultados del ICM resumidos en el CUADRO 3, este es bastante alto en el en comparación con los datos proporcionados por la literatura internacional (17-20), sin existir una diferencia estadísticamente significativa entre ambos grupos.

La atención multidisciplinaria en el hogar, independiente del modelo seguido, ha demostrado mejorar la satisfacción de los 
usuarios y familiares en otras publicaciones (21). Solamente en la pregunta 4 se presentó una diferencia estadísticamente significativa donde un porcentaje menor del grupo de geriatría consideró que tener cubierto los gastos de salud hizo que la experiencia al morir fuera mejor. Es esperable que esto se deba a que probablemente más familias de este grupo no estaban preparadas para la muerte de su familiar. Al analizar el cuarto y último módulo, cuyos resultados se muestran en el CUADRO 4, se documenta, como se mencionó anteriormente, resultados satisfactorios para ambos progrmas. Sin embargo, sí existe una diferencia estadísticamente significativa entre ambos grupos, siendo mucho más satisfactoria la atención del programa de paliativos. Al analizar cada pregunta de forma individual, se documenta diferencias estadísticamente significativas en las preguntas SA 2, SA 3 Y SA 4.

En relación con la calidad de las explicaciones dadas por el equipo y las consutas de emergencia (SA 2 y SA 4), se documenta que en general son muy buenas para ambos grupos, pero la categoría de "mala" sí es estadísticamente significativa mayor en el grupo de geriatría. Con respecto a la pregunta SA 3 y SA 4 (frecuencia de visitas y consulta telefónica) existen diferencias significativas en las categorías de "buena" y "muy buena", teniendo el programa de paliativos una mejor calificación en este aspecto. Estas diferencias probablemente se expliquen por las diferentes características de cada modelo de visitas, ya que, como indican Picado y Sandí (22) el programa de paliativos visita con mayor frecuencia a los pacientes y tiene mayor capacidad de atender urgencias a través de la teleconsulta, algo que ha demostrado mejorar la calidad de atención y la calidad de vida en poblaciones oncológicas (20-25).

Entre las limitaciones del estudio, se destaca que, en proporción, un menor número de pacientes tiene acceso al programa paliativo, por lo que la cantidad de personas entrevistadas que pertenecían a este programa es tres veces menor que la cantidad del modelo de atención geriátrico. Esto puede provocar cierta imprecisión en el ICM de este programa.

Dentro de las fortalezas destacadas del estudio, se encuentra el alto índice de respuesta de los entrevistados lo que genera la suficiente información para obtener un análisis y conclusiones relacionadas a la calidad de muerte en Costa Rica.

\section{CONCLUSIONES}

Según criterio de los cuidadores directos de los pacientes con demencia avanzada del estudio, la calidad de muerte fue muy buena en ambos programas analiziados.

La mayoría de los pacientes de ambos programas murieron con dolor controlado, con poca conciencia de lo que pasaba alrededor, respirando cómodamente, tranquilos, sin miedo a morir, sin preocupaciones por ser una carga para la familia, pasando tiempo con sus amigos y recibiendo afecto de sus seres queridos. La satisfacción de los cuidadores por ambos programas es muy buena siendo significativamente mayor en el programa de paliativos. 


\begin{tabular}{|c|c|c|}
\hline Modalidad de atención & Pacientes visitados & Descripción del programa y servicios ofrecidos \\
\hline Modelo geriátrico & $\begin{array}{l}\text { Enfermedad terminal no } \\
\text { oncológica }\end{array}$ & $\begin{array}{l}\text { Visitas realizadas por equipo multidisciplinario* incluido un } \\
\text { médico geriatra**, } \\
\text { Visitas se realizan cada } 2 \text { o } 3 \text { meses. } \\
\text { Teleconsulta en horario de oficina. } \\
\text { Grupo de capacitación para cuidadores. }\end{array}$ \\
\hline Modelo paliativo & $\begin{array}{l}\text { Enfermedad terminal } \\
\text { oncológica y no } \\
\text { oncológica }\end{array}$ & $\begin{array}{l}\text { Visitas realizadas por equipo multidisciplinario incluido un } \\
\text { médico geriatra y paliativista, visitas se realizan cada } \\
\text { mes o en caso necesario. } \\
\text { Teleconsulta en } 24 / 7 \text { (Por medio de la Fundación Partir } \\
\text { con Dignidad). } \\
\text { Grupo de capacitación para cuidadores. } \\
\text { Grupo de duelo. }\end{array}$ \\
\hline \multicolumn{3}{|c|}{$\begin{array}{l}\text { ^El equipo multidisciplinario está compuesto por un médico, una enfermería, trabajo social, nutrición, terapia del lenguaje, terapia ocupacional, } \\
\text { farmacia, salud mental, odontología y psiquiatría. } \\
\text { ** Las diferencias entre ambos modelos se identifican con letra negrita. } \\
\text { Fuente: Elaboración propia. }\end{array}$} \\
\hline
\end{tabular}

TABLA 2. Costa Rica: Distribución porcentual y promedios de los pacientes del programa crónico y paliativo, según características sociodemográficas e indicadores de salud.

\begin{tabular}{|c|c|c|c|c|c|}
\hline \multicolumn{2}{|l|}{ Variable } & Crónico \% & IC & Paliativo \% & IC \\
\hline \multirow{4}{*}{ Estado civil } & Viudo & 44,8 & 36,$1 ; 53,5$ & 54,1 & 38,$0 ; 70,1$ \\
\hline & Casado & 32,0 & 23,$8 ; 40,2$ & 29,7 & 15,$0 ; 44,5$ \\
\hline & Soltero & 17,6 & 10,$9 ; 24,3$ & 16,2 & 4,$3 ; 28,1$ \\
\hline & Otro & 5,6 & 1,$6 ; 9,6$ * & 0,0 & 0,$0 ; 0,0$ \\
\hline \multirow{2}{*}{ Sexo } & Femenino & 61,6 & 53,$1 ; 70,1$ & 51,4 & 35,$2 ; 67,5$ \\
\hline & Masculino & 38,4 & 29,$9 ; 46,9$ & 48,6 & 32,$5 ; 64,7$ \\
\hline \multirow{2}{*}{ Cuidador principal } & Familiar & 13,8 & 7,$7 ; 19,9$ & 5,4 & 0,$0 ; 12,7$ \\
\hline & Contratado & 86,2 & 80,$1 ; 92,3$ & 94,6 & 87,$3 ; 100,0$ \\
\hline \multirow{2}{*}{ Lugar de fallecimiento } & Casa & 56,0 & 47,$3 ; 64,7^{*}$ & 91,9 & 83,$1 ; 100,0$ \\
\hline & Centro de salud & 44,0 & 35,$3 ; 52,7^{*}$ & 8,1 & 0,$0 ; 17,0$ \\
\hline Úlceras por presión grado 3 o 4 & Sí & 16,0 & 9,$6 ; 22,4$ & 16,2 & 4,$3 ; 28,1$ \\
\hline Sondas enterales de alimentación & Sí & 16,2 & 10,$2 ; 23,4$ & 27,0 & 12,$7 ; 41,3$ \\
\hline Sonda vesical & Sí & 9,6 & 4,$4 ; 14,8$ & 5,4 & 0,$0 ; 13,0$ \\
\hline \multicolumn{2}{|l|}{ Promedio edad } & 85,1 & 83,$8 ; 86,4$ & 84,9 & 82,$3 ; 87,5$ \\
\hline \multicolumn{2}{|c|}{ Promedio edad cuidador } & 54,9 & 52,$6 ; 57,1$ & 52,2 & 48,$2 ; 56,3$ \\
\hline \multicolumn{2}{|l|}{ Promedio Barthel } & 5,2 & 4,$4 ; 6,0$ & 5,3 & 4,$2 ; 6,4$ \\
\hline \multicolumn{6}{|c|}{$\begin{array}{l}\text { IC: Intervalo de confianza al } 95 \% \\
\text { *: Diferencia significativa al } 5 \% \text { en el porcentaje de la categoría medida del programa crónico vs paliativo } \\
\text { Fuente: Elaboración propia. }\end{array}$} \\
\hline
\end{tabular}




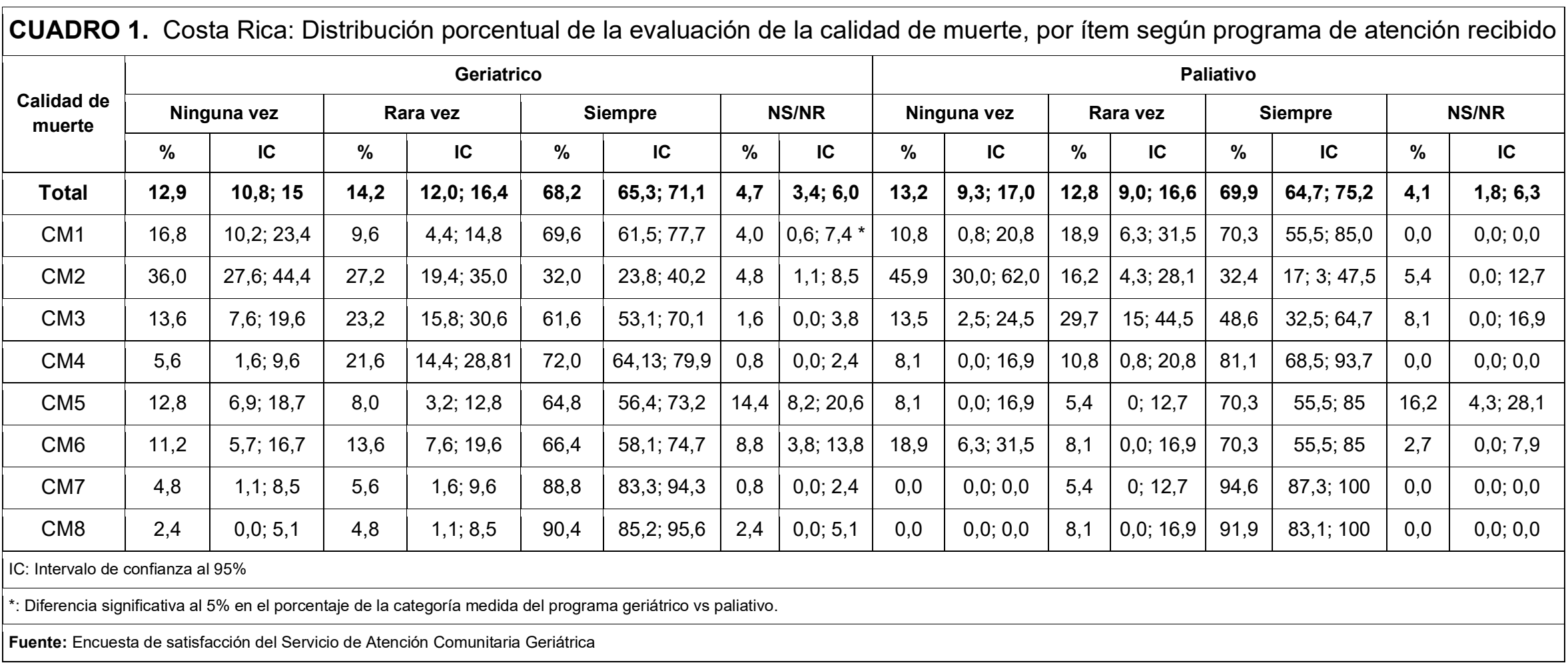


CUADRO 2. Costa Rica: Distribución porcentual de la evaluación de las necesidades básicas, por ítem de medición, según programa de atención recibido.

\begin{tabular}{|c|c|c|c|c|c|c|c|c|c|c|c|c|}
\hline \multirow{3}{*}{$\begin{array}{c}\text { Necesidad } \\
\text { Básica }\end{array}$} & \multicolumn{6}{|c|}{ Crónico } & \multicolumn{6}{|c|}{ Paliativo } \\
\hline & \multicolumn{2}{|r|}{ Sí } & \multicolumn{2}{|r|}{ No } & \multicolumn{2}{|c|}{ NS/NR } & \multicolumn{2}{|r|}{ Sí } & \multicolumn{2}{|r|}{ No } & \multicolumn{2}{|c|}{ NS/NR } \\
\hline & $\%$ & IC & $\%$ & IC & $\%$ & IC & $\%$ & IC & $\%$ & IC & $\%$ & IC \\
\hline Total & 88,4 & 85,$6 ; 91,2$ & 11,0 & 8,$3 ; 13.7$ & 0,6 & 0,$0 ; 1,3$ & 92,6 & 88,$3 ; 96,8$ & 6,8 & 2,$7 ; 10,8$ & 0,7 & 0,$0 ; 2,0$ \\
\hline NB1 & 95,2 & 91,$5 ; 99,0$ & 4,8 & 1,$1 ; 8,5$ & 0,0 & 0,$0 ; 0,0$ & 94,6 & 87,$3 ; 100,0$ & 5,4 & 0,$0 ; 12,7$ & 0,0 & 0,$0 ; 0,0$ \\
\hline NB2 & 81,6 & 74,$8 ; 88,4$ & 17,6 & 10,$9 ; 24,3$ & 0,8 & 0,$0 ; 2,4$ & 91,9 & 83,$1 ; 100,0$ & 8,1 & 0,$0 ; 16,9$ & 0,0 & 0,$0 ; 0,0$ \\
\hline NB3 & 94,4 & 90,$4 ; 98,4$ & 4,8 & 1,$1 ; 8,5$ & 0,8 & 0,$0 ; 2,4$ & 94,6 & 87,$3 ; 100,0$ & 5,4 & 0,$0 ; 12,7$ & 0,0 & 0,$0 ; 0,0$ \\
\hline NB4 & 82,4 & 75,$7 ; 89,1$ & 16,8 & 10,$2 ; 23,4$ & 0,8 & 0,$0 ; 2,4$ & 89,2 & 79,$2 ; 99,2$ & 8,1 & 0,$0 ; 16,9$ & 2,7 & 0,$0 ; 7,9$ \\
\hline \multicolumn{13}{|c|}{ IC: Intervalo de confianza al 95\% } \\
\hline \multicolumn{13}{|c|}{ *: Diferencia significativa al $5 \%$ en el porcentaje de la categoría medida del programa geriátrico vs paliativo. } \\
\hline
\end{tabular}

CUADRO 3. Costa Rica: Índice de calidad de muerte según programa de atención recibido

\begin{tabular}{|c|c|c|c|c|}
\hline \multirow{2}{*}{ Índice } & \multicolumn{2}{|c|}{ Crónico } & \multicolumn{2}{|c|}{ Paliativo } \\
\hline & Promedio & IC & Promedio & IC \\
\hline Total & 8,79 & 8,$66 ; 8,92$ & 9,09 & 8,$86 ; 9,32$ \\
\hline ICM1 & 9,19 & 8,$89 ; 9,49$ & 9,43 & 8,$88 ; 9,98$ \\
\hline ICM2 & 9,48 & 9,$21 ; 9,75$ & 9,65 & 9,$33 ; 9,97$ \\
\hline ICM3 & 9,48 & 9,$20 ; 9,76$ & 9,72 & 9,$39 ; 10,00$ \\
\hline ICM4 & 8,00 & 6,$36 ; 9,64^{*}$ & 10,00 & 10,$00 ; 10,00$ \\
\hline ICM5 & 9,36 & 9,$05 ; 9,67$ & 9,44 & 8,$87 ; 10,00$ \\
\hline ICM6 & 7,90 & 7,$33 ; 8,47$ & 8,19 & 7,$24 ; 9,14$ \\
\hline ICM7 & 8,44 & 7,$97 ; 8,91$ & 8,58 & 7,$66 ; 9,50$ \\
\hline ICM8 & 8,29 & 7,$79 ; 8,79$ & 8,69 & 7,$88 ; 9,50$ \\
\hline ICM9 & 8,98 & 8,$58 ; 9,38$ & 9,32 & 8,$61 ; 10,00$ \\
\hline ICM10 & 8,09 & 7,$54 ; 8,64$ & 8,38 & 7,$21 ; 9,55$ \\
\hline ICM11 & 8,03 & 7,$46 ; 8,60$ & 8,43 & 7,$35 ; 9,51$ \\
\hline ICM12 & 9,24 & 8,$89 ; 9,59$ & 9,66 & 9,$20 ; 10,00$ \\
\hline \multicolumn{5}{|c|}{ IC: Intervalo de confianza al 95\% } \\
\hline \multicolumn{5}{|c|}{ *: Diferencia significativa al $5 \%$ en el procentaje de la categoría medida del programa crónico vs paliativo } \\
\hline
\end{tabular}


CUADRO 4. Costa Rica: Distribución porcentual del nivel de satisfacción, por ítem medido, según programa de atención recibido.

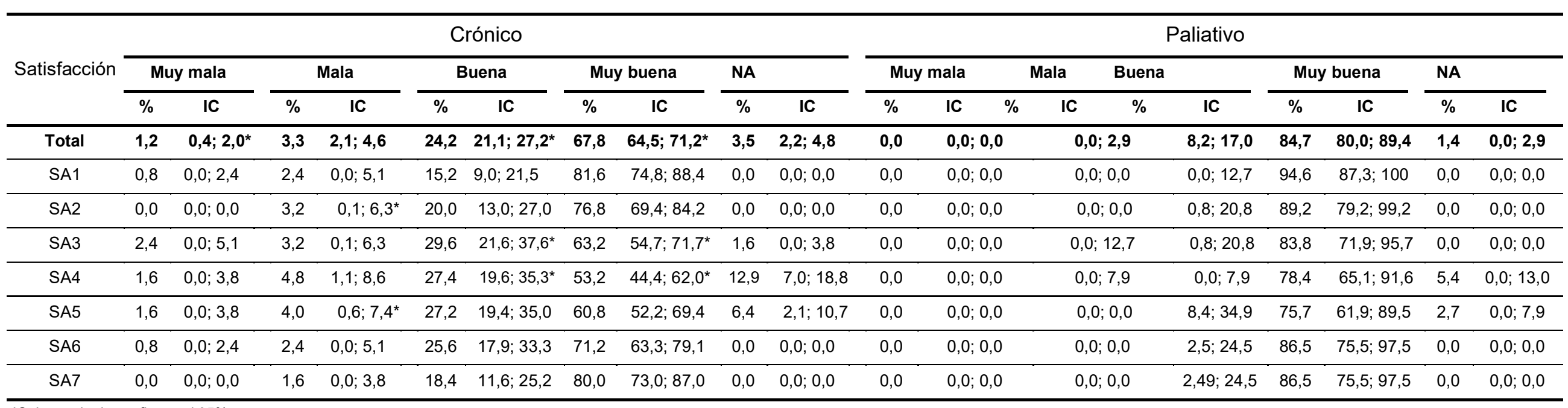

IC: Intervalo de confianza al $95 \%$

*: Diferencia significativa al $5 \%$ en el porcentaje de la categoría medida del programa crónico vs paliativo

Fuente: Encuesta de satisfacción del Servicio de Atención Comunitaria Geriátrica 


\section{REFERENCIAS}

1. United Nations, Department of Economic and Social Affairs, Population Division (2015). World Population Ageing 2015 (ST/ESA/SER.A/390).

2. Alzheimer's Disease International: World Alzheimer Report 2015 [citado 13 setiembre 2020]. Disponible en: https://www.alz.co.uk/research/WorldAlzheimerReport2015.pdf.

3. Van der Steen J. T. Dying with dementia: what we know after more than a decade of research. Journal of Alzheimer's disease : JAD. 2010; 22 (1): 37-55. https://doi.org/10.3233/JAD-2010-100744.

4. Merel S, et al. Palliative Care in Advanced Dementia Clin Geriatr Med. 2014; 30 (3):469-92.

5. Mitchell Susan. The Course of avance of dementia. N. Engl Med. 2014; 16: 361

6. Mitchell, S. Care of patients with advanced dementia UpToDate [internet] 2019 [citado 20 abr. 2019] Disponible en: https://www.uptodate.com/contents/care-of-patients-with-advanced-dementia\#H423254626

7. van der Plas, A. G., Oosterveld-Vlug, M. G., Pasman, H. R., \& Onwuteaka-Philipsen, B. D. (2017). Relating cause of death with place of care and healthcare costs in the last year of life for patients who died from cancer, chronic obstructive pulmonary disease, heart failure and dementia: A descriptive study using registry data. Palliative medicine, 31(4), 338-345. https://doi.org/10.1177/0269216316685029.

8. Radbruch, L, Payne, S and Board of Directors of the EAPC. White paper on standards and norms for hospice and palliative care in Europe, part 1: recommendations from the European Association for Palliative Care. Eur J Palliat Care 2009; 16: 278-289. https://eapcresearchcongress2020.eu/wp-content/uploads/2020/11/White-Paper-onstandards-and-norms-for-hospice-and-palliative-care-in-Europe.pdf

9. Brian Cassel, J., Kerr, K. M., McClish, D. K., Skoro, N., Johnson, S., Wanke, C., \& Hoefer, D. (2016). Effect of a Home-Based Palliative Care Program on Healthcare Use and Costs. Journal of the American Geriatrics Society, 64(11), 2288-2295. https://doi.org/10.1111/jgs.14354.

10. Bartley, MM. Dementia Care at End of Life: Current Approaches. Curr Psychiatry Rep. 2018. Jun 23; 20 (7): 507. https://doi.org/10.1007/s11920-018-0915-x

11. Ellen E. Lee, Beverly Chang, Steven Huege, Jeremy Hirst. A Complex Clinical Intersection: Palliative Care in Patients with Dementia, Am J Geriatr Psychiatry. 2017; 26(2): 224-234. https://doi.org/10.1016/j.jagp.2017.06.015

12. Stewart JT, Schultz SK. Palliative Care for Dementia. Psychiatr Clin North Am. 2018 Mar;41(1):141-151. https://pubmed.ncbi.nlm.nih.gov/29412842/

13. Yumoto, Yoshie \& Kernohan, Wgeorge \& Morioka, Noriko \& Ogata, Yasuko. International reflections on caring for people with advanced dementia. Public Health Nurs. 2018; 36:192-198. https://doi.org/10.1111/phn.12572

14. Picado-Ovares, JE., Hernández-Zamora, P., \& Escobar-Salas. A. Atención Comunitaria Geriátrica. En Tratado de Geriatría y Gerontología (2. ${ }^{a}$ ed. San José: EDNASS; 2016. 187-207.

15. Pérez, P, et al. Validation of the Spanish Version of the Quality of Dying and Death Questionnaire (QODD-ESP) in a Home-Based Cancer Palliative Care Program and Development of the QODD-ESP-12. Journal of Pain and Symptom Management. 2017; 53 (6): 1042-1049.e3. https://doi.org/10.1016/.jpainsymman.2017.02.005 
16. Zimmermann C, Riechelmann R, Krzyzanowska M, Rodin G, Tannock I. Effectiveness of Specialized Palliative Care: A Systematic Review. JAMA. 2008;299(14):1698-1709. https://doi.org/10.1001/jama.299.14.1698

17. Brumley, R., Enguidanos, S., Jamison, P., Seitz, R., Morgenstern, N., Saito, S., Mcllwane, J., Hillary, K., \& Gonzalez, J. (2007). Increased satisfaction with care and lower costs: results of a randomized trial of in-home palliative care. Journal of the American Geriatrics Society, 55(7), 993-1000. https://doi.org/10.1111/j.15325415.2007.01234.x

18. Curtis, J. R., Patrick, D. L., Engelberg, R. A., Norris, K., Asp, C., \& Byock, I. A measure of the quality of dying and death. Initial validation using after-death interviews with family members. Journal of pain and symptom management, 2002; 24 (1): 17-31. https://doi.org/10.1016/s0885-3924 (02) 00419-0.

19. Hales, S., Zimmermann, C., \& Rodin, G. Review: the quality of dying and death: a systematic review of measures. Palliative medicine. (2010); 24(2): 127-144. https://doi.org/10.1177/0269216309351783.

20. Gomes, B., Calanzani, N., Curiale, V., McCrone, P., \& Higginson, I. J. (2013). Effectiveness and cost-effectiveness of home palliative care services for adults with advanced illness and their caregivers. The Cochrane database of systematic reviews, (6), CD007760. https://doi.org/10.1002/14651858.CD007760.pub2.

21. Wen, J., \& Schulman, K. A. (2014). Can team-based care improve patient satisfaction? A systematic review of randomized controlled trials. PloS one, 9(7), e100603. https://doi.org/10.1371/journal.pone.0100603.

22. Picado-Ovares JE, Sandí Jirón A. Perfil del paciente con demencia del programa de atención domiciliaria geriátrica del Hospital Nacional de Geriatría y Gerontología Doctor Raúl Blanco Cervantes - San José, Costa Rica. Rev.méd.sinerg. [Internet]. 1 de noviembre de 2020 [citado 4 de diciembre de 2020];5(11):e608. Disponible en: https://revistamedicasinergia.com/index.php/rms/article/view/608.

23. Bradford, N., Armfield, N. R., Young, J., \& Smith, A. C. (2013). The case for home based telehealth in pediatric palliative care: a systematic review. BMC palliative care, 12, 4. https://doi.org/10.1186/1472-684X-12-4.

24. Kitamura, C., Zurawel-Balaura, L., \& Wong, R. K. (2010). How effective is video consultation in clinical oncology? A systematic review. Current oncology (Toronto, Ont.), 17(3), 17-27. https://doi.org/10.3747/co.v17i3.513.

25. Laila, M., Rialle, V., Nicolas, L., Duguay, C., \& Franco, A. (2008). Videophones for the delivery of home healthcare in oncology. Studies in health technology and informatics, 136, 39-44. https://pubmed.ncbi.nlm.nih.gov/18487705/ 